\title{
First step in the nuclear inverse Kohn-Sham problem: From densities to potentials
}

\author{
G. Accorto \\ Dipartimento di Fisica “Aldo Pontremoli”, Università degli Studi di Milano, Via Celoria 16, 20133 Milano, Italy \\ and Department of Physics, Faculty of Science, University of Zagreb, Zagreb, Croatia \\ P. Brandolini, F. Marino, A. Porro $\odot$, and A. Scalesi $\odot$ \\ Dipartimento di Fisica "Aldo Pontremoli”, Università degli Studi di Milano, Via Celoria 16, 20133 Milano, Italy \\ G. Colò ${ }^{*}$ and X. Roca-Maza $\odot^{\dagger}$ \\ Dipartimento di Fisica "Aldo Pontremoli", Università degli Studi di Milano, Via Celoria 16, 20133 Milano, Italy \\ and INFN, Sezione di Milano, Via Celoria 16, 20133 Milano, Italy \\ E. Vigezzi \\ INFN, Sezione di Milano, Via Celoria 16, 20133 Milano, Italy
}

(Received 8 August 2019; revised manuscript received 22 November 2019; accepted 10 February 2020; published 28 February 2020)

\begin{abstract}
Nuclear density functional theory (DFT) plays a prominent role in the understanding of nuclear structure, being the approach with the widest range of applications. Hohenberg and Kohn theorems warrant the existence of a nuclear energy density functional (EDF), yet its form is unknown. Current efforts to build a nuclear EDF are hindered by the lack of a strategy for systematic improvement. In this context, alternative approaches should be pursued and, so far, an unexplored avenue is that related to the inverse DFT problem. DFT is based on the one-to-one correspondence between Kohn-Sham (KS) potentials and densities. The exact EDF produces the exact density, so that from the knowledge of experimental or ab initio densities one may deduce useful information through reverse engineering. The idea has already been proved to be useful in the case of electronic systems. The general problem should be dealt with in steps, and the objective of the present work is to focus on testing algorithms to extract the Kohn-Sham potential within the simplest ansatz from the knowledge of the experimental neutron and proton densities. We conclude that, while robust algorithms exist, the experimental densities present some critical aspects. Finally, we provide some perspectives for future works.
\end{abstract}

DOI: 10.1103/PhysRevC.101.024315

\section{INTRODUCTION}

Density functional theory (DFT) has become gradually one of the best tools of choice for the study of nuclear structure $[1,2]$, trying to follow the path that led to the success of electronic DFT $[3,4]$. There are analogies and differences between the two cases. One can expect that building an energy density functional (EDF) for nuclei is harder than doing the same for electronic systems, in keeping with the more involved, and less well known, underlying nucleon-nucleon $(\mathrm{NN})$ interaction. This interaction is strongly spin- and isospin-dependent, while momentum-dependent, spin-orbit, and tensor terms are far from being negligible and there are also three-body (NNN) components; all this is at variance with the Coulomb case.

DFT is grounded in the Hohenberg-Kohn theorems (HKTs), stating that a universal EDF must exist and yet not

\footnotetext{
*gianluca.colo@mi.infn.it

†xavier.roca.maza@mi.infn.it

‡enrico.vigezzi@mi.infn.it
}

providing any guidance on how to build its terms [5]. The most used and well-established nuclear EDFs like the Skyrme and Gogny ones (we do not discuss covariant functionals which, though very successful, are outside our scope here) include terms that have their origin in central two-body forces and have a form proportional to the square of the total number density $\left(\rho^{2}\right)$ and repulsive terms which depend on a larger power of $\rho$ to mimic short-range repulsion, besides the terms that have been mentioned in the previous paragraph and account for spin forces, spin-orbit forces, etc. They contain parameters that are fitted on experimental properties of selected nuclei, can be dubbed as phenomenological, and lack from the beginning a clear mechanism for systematic improvement.

Recently, several groups have undertaken important steps to build more general EDFs, in which one starts from $\rho$ dependent terms, and include other terms that depend on gradients $\vec{\nabla} \rho$ up to a given order (see [6], as well as [7] and references therein). The systematic construction of all possible densities and their gradients has been described in the past $[8,9]$, together with the systematic classification of all possible terms that should enter a nuclear EDF [10]. These 
terms are all the scalar quantities that can be built out of densities and that are invariant under parity, time-reversal, rotational, translational, and isospin transformations. Obviously, the number of such terms can be very large, and fitting too general EDFs may become either technically prohibitive or impractical due to the lack of experimental input, or both.

Other attempts have been made to derive the nuclear EDFs from fundamental approaches. In this regard, general ideas and perspectives can be found in Ref. [11]. Whereas in Refs. [12-14] attempts have been made to derive nonrelativistic EDFs from Brückner-Hartree-Fock calculations in uniform matter, a hybrid approach has been followed in Refs. [15-17], in which the long-range, pion exchange-like part of the EDF has been derived from chiral forces and short-range coupling constants are left to be fitted against phenomenological data. While trying to derive EDFs from $a b$ initio theories, or to generalize their structure, may produce some breakthroughs, perhaps new mathematical or computational techniques are also worth attempting.

The present work is based on the Kohn-Sham (KS) realization of the HKTs [18]. We define the direct problem as the one in which, given a KS functional and the associated effective potential, we deduce the density that can be compared to experiment as well as the total ground-state energy. We define the inverse problem as the one in which, starting from a given (supposedly exact) density, we deduce the effective KS potential. Solving the inverse problem is obviously appealing as it can constrain the phenomenological KS potential and the phenomenological EDF at its basis. In the case of electronic systems there have been several attempts to attack the inverse KS (IKS) problem. In this work, we closely follow some of the inversion methods reviewed in Ref. [19], in which some basic concepts and techniques are discussed in detail. Hence, the current work is motivated by the idea that if the density is the basic variable to describe fermionic systems, as guaranteed by the HKTs, the nuclear densities should contain, in principle, all the relevant information to constrain the nuclear EDF.

The structure of the present paper is as follows. In Sec. II, we introduce the KS realization of the HKTs as well as the inverse problem, highlighting the specific aspects of the nuclear case as we have just mentioned. In Sec. III, we describe the two adopted computational approaches. In Sec. IV, we test the numerical methods presented in Sec. III by using theoretical densities generated from a mean-field approach. In Sec. V, we deduce from experiment the KS potential for protons in ${ }^{40} \mathrm{Ca}$ and for neutrons and protons for ${ }^{208} \mathrm{~Pb}$. Our conclusions will be presented in Sec. VI, together with some future perspectives for this work.

\section{INVERSE KOHN-SHAM PROBLEM}

As is well known, the KS method is the most practical way to implement DFT. This method is based on the idea that solving the problem of the interacting system under study is equivalent to solving a system of independent particles subject to an effective potential, provided the density is the same. In fact, the ground-state number density can be expressed as the sum over a number $N_{\text {orb }}$ of KS single-particle orbitals $\phi_{i}$, namely

$$
\rho(\vec{r})=\sum_{i}^{N_{\text {orb }}}\left|\phi_{i}(\vec{r})\right|^{2}
$$

In addition, it is assumed that the kinetic energy $T$ has the same form as in the independent particle case. Then, the EDF is written as

$$
E[\rho]=\sum_{i} \int d^{3} r \phi_{i}^{*}(\vec{r})\left(-\frac{\hbar^{2}}{2 m}\right) \nabla^{2} \phi_{i}(\vec{r})+F[\rho],
$$

and its minimization leads to the well-known Kohn-Sham equations of the type

$$
\left(-\frac{\hbar^{2}}{2 m} \nabla^{2}+\frac{\delta F[\rho]}{\delta \rho}\right) \phi_{i}(\vec{r})=\varepsilon_{i} \phi_{i}(\vec{r}),
$$

where $\varepsilon_{i}$ are the so-called Kohn-Sham eigenvalues. The quantity $\frac{\delta F[\rho]}{\delta \rho}$ plays the role of an effective potential and will be hereafter denoted by $U[\rho]$. If $F$ is assumed, $U$ is given and the density can be found. In this context, the IKS problem consists of reversing the procedure and deriving the effective potential $U[\rho]$ given the knowledge of $\rho$. As already mentioned, in the present work only neutron and proton densities will be considered. That is, we will assume that the $\mathrm{KS}$ potential is a function of space coordinates through the dependence on the local densities only. This potential is determined except for a constant shift. In KS-DFT, eigenvalues and orbitals are auxiliary quantities which, strictly speaking, have no physical meaning. The exception is the energy of the last occupied orbital, that coincides with the ionization energy in atomic systems [20,21] or with the neutron/proton separation energy in nuclear systems. This provides a unique way to set the absolute value of the KS potential, that goes to zero at large distances as it should.

In the literature, one finds several formulations of the IKS problem for the case of electronic systems, (see, e.g., [19] for a recent review and [22] for early references). The two approaches that we discuss below were originally introduced in Refs. [19,23]. The IKS problem has been solved in the case of the He atom, where an exact analytic solution for wave functions and densities is available [24], and this has allowed testing various approximate methods [25]. Among recent papers, we also would like to mention Refs. [26,27], that deal with the time-dependent inverse problem, and Ref. [28], that shows the connection between different IKS strategies. References [29,30] should also be highlighted. This list is not meant to be exhaustive. In nuclear physics, some groups have tried to deduce a heavy-ion potential from time-dependent Hartree-Fock calculations [31]. Anyway, to the best of our knowledge, ours is the first attempt to address the IKS problem in the nuclear case. Therefore, we discuss some specific issues in what follows.

Is the IKS problem well posed? One finds ample discussion in the literature regarding the question of whether or not the IKS is well-posed, according to the definition given by Hadamard [32]. As reported in [19], according to Hadamard a problem is well posed if a solution exists, it is unique, and it depends continuously on the data. If any of these three 
properties is violated then the problem is ill posed. We miss a formal proof of the fact that IKS is well posed, except for the case of discretized systems [33], but this does not mean that the IKS problem is necessarily ill posed. Still, its numerical solution is a very delicate matter. From a theoretical point of view, the existence of a Kohn-Sham potential for a given experimental density is not guaranteed, although the uniqueness of the solution is guaranteed if a method converges (see, e.g., the discussion of not $v$-representable densities in [34]). Furthermore, uncertainties and lack of completeness in the input density data can lead to violations of the Hadamard conditions that we have just mentioned. Some of the pathologies inherent to experimental nuclear densities will be discussed in Sec. V.

$E D F$ from $K S$ potential. Going one step back from the effective potential $U[\rho(\vec{r})]$ to the functional $F[\rho(\vec{r})]$ is also possible. In electronic systems (cf., e.g., Ref. [35]), it has been shown with concrete examples that the exchange-correlation functional $E_{\mathrm{xc}}$ can be reconstructed starting from the knowledge of the associated exchange-correlation potential $v_{\mathrm{xc}}$. The basic formula to achieve this goal was introduced by van Leeuwen and Baerends in Ref. [36], and reads

$$
E_{\mathrm{xc}}[\rho]=\int_{0}^{1} d t \int d^{3} r v_{\mathrm{xc}}\left[\rho_{t}\right] \frac{\partial \rho_{t}}{\partial t} .
$$

Here, $\rho_{t}$ is a continuously parametrized density such that $E_{\mathrm{xc}}\left[\rho_{0}\right]=0$ and $\rho_{1}=\rho(\vec{r})$. This equation implies the knowledge of the potential along a path of densities which, as we mentioned above, is not obtainable from any experimental input but only from $a b$ initio calculations. For example, calculations of systems of few nucleons confined in an external harmonic oscillator potential (the so-called neutron drops) may provide a path of densities as the confining potential can be varied continuously. We shall try to elaborate on this in the conclusions.

Different types of nuclear densities. In the nuclear case, various kinds of densities must be used for a realistic EDF. In fact, the classification of all possible local densities that may (at least in principle) enter a local EDF, and the way to build them, is discussed in detail in Refs. [1,8,9]. Consequently, our current inversion of Eq. (3) is intended as a first step towards more realistic applications, as we employ here neutron and proton number densities alone. We shall need, in future, to formulate the inverse $\mathrm{KS}$ problem also in terms of other relevant nuclear densities. In this case, the implementation will require the input from $a b$ initio theories. We give just two examples here. If we wish to include gradient terms in our considerations, we could extract the density dependence from uniform matter and formulate the IKS in finite nuclei to obtain the gradient terms only. If we wish to include spin terms, we can formulate the IKS having the spin density of odd nuclei from ab initio calculations. We shall come back to this in the conclusions.

Laboratory density and intrinsic density. A fundamental difference between DFT in finite electronic systems and nuclei consists in the fact that in the former case the fixed ion positions constrain the shape of the system in the laboratory frame. In nuclei, that are self-bound systems, this is not the case. The usual HK theorem, as argued by several authors [37], is formulated for the laboratory density, while experiments on nuclei probe the intrinsic density (relative to the nuclear center of mass). Nonetheless, it has been proved that, given an arbitrary Hermitian operator $\hat{Q}$, one can build an energy functional depending on $Q(\vec{r}) \equiv\langle\hat{Q}(\vec{r})\rangle$ that is universal in the HK sense and has its minimum at the correct value of $Q$ with the correct energy (see for example Sec. II of Ref. [37] as well as Refs. [38,39]). In this respect, being the intrinsic density an Hermitian operator, one can replace the laboratory density with the intrinsic density in the HK theorem $[40,41]$.

\section{INVERSE KOHN-SHAM PROBLEM: TWO DIFFERENT METHODS}

We start from Eq. (3) and assume that the effective potential $U[\rho]$ is only position dependent, in keeping with the KS ansatz described in Sec. II. Thus, within this ansatz, nonlocal effects and the spin-orbit potential are not explicitly taken into account. The latter approximation should not impact much the KS potential since spin-orbit effects are not expected to markedly change the KS orbitals. Consequently, from Eq. (1), the density should be almost untouched. Nonlocality could be accounted for via gradients of the density but, as we have said above, we leave this for future improvements. As for the Coulomb interaction between protons, we implicitly assume a local form within $U[\rho]$ in Eq. (3). This is known to work well for the description of the total binding energy and density in nuclei $[42,43]$.

We use two different methods to extract the KS potential $U[\rho]$ from the neutron and proton densities. The first one is based on an iterative procedure and was introduced by van Leeuwen and Baerends [23]; it will be called the vLB method. The second method consists instead of the constrained minimization of the kinetic energy $T$, in the spirit of KS that introduces an auxiliary system of independent particles with the same density as the system under study; this method will be called the constrained variational, or CV, method. In choosing the notation vLB and CV, we follow Ref. [19]. In both cases we will use $\tilde{\rho}$ to denote the target density, that is, the density to be reproduced. We will restrict ourselves to doubly magic, spherical systems.

\section{A. The vLB method}

The aim of this procedure is to bring the calculated density as close as possible to the given target density by iteration. That is, by starting from an initial guess of $U[\rho]$, one implements the vLB algorithm [23] to calculate the new $U[\rho]$ and repeats it until $U[\rho]$ is stable. The derivation of the vLB algorithm is very simple so we outline it in what follows.

Let us start by writing the direct formulation of the KS equation (3) in spherical symmetry,

$$
\left[-\frac{\hbar^{2}}{2 m} \frac{d^{2}}{d r^{2}}+\frac{\hbar^{2} l(l+1)}{2 m r^{2}}+U(r)\right] u_{i}(r)=\varepsilon_{i} u_{i}(r),
$$

where $u_{i}(r)$ are the reduced radial wave functions and $U(r) \equiv$ $U[\rho(r)]$ is the effective Kohn-Sham potential that has been introduced already. In the case of spherical nuclei, $i$ stands for $n, l, j$, which denote the principal quantum number, the orbital 
angular momentum, and the total angular momentum. The full wave function reads $\phi_{i}(\vec{r}) \equiv \frac{u_{n l j}(r)}{r}\left[Y_{l}(\theta, \phi) \otimes \chi_{1 / 2}\right]_{j m}$, where $m$ denotes the projection of the angular momentum on the $z$ axis. We do not use specific notations for protons and neutrons as the iterative procedure is carried out independently for the two species. As already stressed, we assume here that the spinorbit potential does not change significantly the shape of the radial wave function, so that $u_{i}(r)$ is effectively the same for the spin-orbit partners $j=l+1 / 2$ and $j=l-1 / 2$.

The boundary conditions at the origin associated with Eq. (5) are well known,

$$
\begin{aligned}
& \lim _{r \rightarrow 0} u(r)=r^{l+1}, \\
& \lim _{r \rightarrow 0} u^{\prime}(r)=(l+1) r^{l},
\end{aligned}
$$

and these allow one to solve the direct Kohn-Sham equation (5) by means of a shooting algorithm. Thus, we find the energy eigenvalues and the eigenfunctions and derive the density $\rho(r)$ as

$$
\rho(r)=\frac{1}{4 \pi r^{2}} \sum_{i=0}^{N_{\mathrm{orb}}} n_{i} u_{i}^{2}(r)
$$

where $n_{i}$ is the occupation factor of the orbital $i$. In principle, $n_{i}$ could be taken as a fractional particle number (cf. Ref. [20]). However, we assume here $n_{i}=2 j+1$ since we limit ourselves to closed shell nuclei. We have checked that, not having implemented any spin-orbit effect in Eq. (5), working within the uncoupled $l$ and $s$ or coupled $j=l+s$ scheme provides identical results.

The vLB inverse algorithm introduced in Ref. [23] (as well as a slightly different algorithm [22] that is not discussed here) can be obtained by algebraic manipulation of Eq. (5). Specifically, by multiplying Eq. (5) by $n_{i} u_{i}(r)$ on both sides, summing over $i$, and dividing by $4 \pi r^{2} \rho(r)$, one finds

$$
U(r)=\frac{1}{4 \pi r^{2} \rho(r)} \sum_{i=0}^{N_{\text {orb }}}\left[n_{i} u_{i}(r)\left(\frac{\hbar^{2}}{2 m} \frac{d^{2}}{d r^{2}}-U_{l}\right) u_{i}+\varepsilon_{i} n_{i} u_{i}^{2}\right]
$$

where $U_{l}$ is a shorthand notation for the centrifugal potential. Now we need to define the iterative process from that equation. Denoting iteration numbers by superscripts, a guess for the new potential $U^{(k+1)}$ can be obtained by substituting $\rho$ with $\tilde{\rho}(r)$ in the denominator at the right-hand side of Eq. (8), and realizing that the rest of the right-hand side in the same equation corresponds to the potential times the density determined in the previous iteration, or $4 \pi r^{2} \rho^{(k)}(r) U^{(k)}$. That is,

$$
\begin{aligned}
U^{(k+1)}(r)= & \frac{1}{4 \pi r^{2} \tilde{\rho}(r)} \sum_{i=0}^{N_{\text {orb }}}\left[n_{i} u_{i}^{(k)}(r)\left(\frac{\hbar^{2}}{2 m} \frac{d^{2}}{d r^{2}}-U_{l}\right) u_{i}^{(k)}\right. \\
& \left.+\varepsilon_{i} n_{i}\left(u_{i}^{(k)}\right)^{2}\right]=\frac{\rho^{(k)}(r)}{\tilde{\rho}(r)} U^{(k)}(r)
\end{aligned}
$$

It is important to note that Eq. (9) has a simple meaning. In regions where the density at the $k$ th step is larger than the target density the potential is increased in absolute value, and vice versa. This makes sense for repulsive potentials as in the electronic case, but, in the case of the attractive potentials needed in nuclear physics, the opposite should happen. To avoid this problem, we have adopted a modified algorithm proposed in Ref. [19]:

$$
U^{k+1}(r)=U^{(k)}(r)+\gamma \frac{\rho^{(k)}(r)-\tilde{\rho}(r)}{\tilde{\rho}(r)} .
$$

This algorithm works equally well for attractive and repulsive potentials and is invariant under an arbitrary shift of the potential, providing a convenient numerical alternative. Furthermore, we have found that the simple choice $\gamma=1$ $\mathrm{MeV}$ leads to consistent results.

In short, our choice has been that of assuming a starting potential $U^{(k=1)}(r)$, calculating the eigenstates $u^{(k=1)}(r)$ from Eq. (5) together with the density (7), then finding a new potential by applying Eq. (10). The procedure is repeated until convergence. The convergence condition used to stop the iterative procedure is set in terms of the absolute variation of the potential, that is,

$$
\Delta U^{(k)} \equiv \max _{r}\left|U^{(k+1)}(r)-U^{(k)}(r)\right|<\alpha .
$$

Remarkably, despite its simplicity, the algorithm has proved to be robust enough to converge to the same results, assuming $U^{(k=1)}(r)$ to be equal either to a realistic Woods-Saxon potential or to a simple constant.

\section{B. The CV method}

In the CV method, the IKS problem is formulated as a variational problem. The formulation is in keeping with the $\mathrm{KS}$ ansatz, that asserts that for any interacting Fermi system one can always postulate an independent particle system with the same density. Accordingly, in the CV method one writes down the kinetic energy functional of the $N$ fermions with the purpose of minimizing it. Therefore, in what follows, we shall use the name "objective functional" for the expectation value of the kinetic energy associated with the wave function of $N$ independent particles as in Eq. (2). The minimization is subject to the following constraints:

(1) the single-particle orbitals $\phi_{i}(\vec{r})$ must be orthonormal;

(2) the density of the system $\rho(\vec{r})$ must be equal to the target density $\tilde{\rho}(\vec{r})$, for each value of $\vec{r}$.

Let us start from the same assumptions as in the previous subsection. We assume that we have $N_{\text {orb }}$ single-particle states and that each of them has occupancy $n_{i}$. We prefer here to write the equation without going immediately to the spherically symmetric case, as the CV method is more apt for the generalization to the case in which this symmetry is totally or partially broken. The objective functional reads

$$
T_{s}\left[\left\{\phi_{i}\right\}\right]=\sum_{i=1}^{N_{\text {orb }}} n_{i} \int d^{3} r \phi_{i}^{*}(\vec{r})\left(-\frac{\hbar^{2}}{2 m}\right) \nabla^{2} \phi_{i}(\vec{r}) .
$$

The orthonormality of the orbitals is the first constraint and is expressed by

$$
G_{i j}\left[\left\{\phi_{i}\right\}\right]=\int d^{3} r \phi_{i}^{*}(\vec{r}) \phi_{j}(\vec{r})=\delta_{i j}
$$


The equality of the density to the target density represents the second constraint [see Eq. (14)]. We introduce Lagrange multipliers that correspond to these constraints. Those associated with the constraints (13) are indicated as $\varepsilon_{i j}$, while we write the constraint associated with the density as $U(\vec{r})$. The constrained minimization of the objective functional is rewritten as the free minimization of the functional that includes the Lagrange multipliers, which is named the cost functional [44]. This cost functional reads

$$
\begin{aligned}
J\left[\left\{\phi_{i}\right\} ; U(\vec{r}),\left\{\varepsilon_{i j}\right\}\right]= & T_{s}\left[\left\{\phi_{i}\right\}\right]+\int d^{3} r U(\vec{r}) \rho(\vec{r}) \\
& -\sum_{i=1}^{N_{\text {orb }}} \sum_{j=1}^{i} \varepsilon_{i j} \int d^{3} r \phi_{i}^{*}(\vec{r}) \phi_{j}(\vec{r}),
\end{aligned}
$$

and the $\mathrm{CV}$ method consists of solving the equation

$$
\delta J\left[\left\{\phi_{i}\right\} ; U(\vec{r}),\left\{\varepsilon_{i j}\right\}\right]=0 .
$$

It is important to note that, as explained on p. 146 of Ref. [45], the optimization of the cost functional (15) leads to a nondiagonal form of the $\mathrm{KS}$ equations. In other terms, the wave functions $\phi_{i}$ that are solutions of (15) are not eigenfunctions of the KS equations, and $\varepsilon_{i i}$ do not correspond to the KS eigenvalues. Nevertheless, the set $\phi_{i}$ and the matrix $\varepsilon_{i j}$ are related to the orbitals that solve the KS equation and to the diagonal matrix with the KS eigenvalues, respectively, by a unitary transformation. At the same time, the very form of Eq. (14) tells us that the extracted Lagrange multiplier $U(\vec{r})$ is the Kohn-Sham potential, up to a constant shift. In this respect, the $\mathrm{CV}$ method is a direct formulation of the IKS.

Minimizing the cost functional $J\left[\left\{\phi_{i}\right\} ; U(\vec{r}),\left\{\varepsilon_{i j}\right\}\right]$ means to find the set of orbitals $\phi_{i}$, defined on a given domain, that gives the minimum value for the functional respecting the two constraints at the same time. Hence, an unrestricted three-dimensional formulation is technically involved. In order to compare to our results obtained with the vLB method presented in Sec. III A, we have limited ourselves to the case of spherical nuclei, as already discussed, so that the problem becomes one-dimensional, and the orbitals depend only on $r$. In spherical symmetry, Eq. (14) becomes

$$
\begin{aligned}
J\left[\left\{u_{j}\right\} ; U(r),\left\{\epsilon_{i j}\right\}\right]= & T_{s}\left[\left\{u_{j}\right\}\right]+4 \pi \int_{0}^{\infty} U(r) \rho(r) r^{2} d r \\
& -\sum_{i=1}^{N_{\text {orb }}} \sum_{j=1}^{i} \epsilon_{i j} \delta_{l_{i} l_{j}} \delta_{j_{i} j_{j}} \int_{0}^{\infty} u_{i}(r) u_{j}(r) d r .
\end{aligned}
$$

The CV method has been implemented following some important modifications suggested in Ref. [19]. First, a new set of variables, viz., the rescaled orbitals $f_{i}(r)$ that are defined by

$$
u_{i}(r)=\sqrt{4 \pi \tilde{\rho}(r)} r f_{i}(r),
$$

have been introduced. The rationale behind this substitution is that we expect that $u_{i}^{2}(r) \approx 4 \pi r^{2} \tilde{\rho}(r)$, so that the rescaling will produce new functions $f_{i}(r)$ of the order of $\approx 1$, characterized by a milder behavior as compared to $u_{i}(r)$. This helps in reducing the rounding errors that appear when operating with quantities of different orders of magnitude. The CV iterative procedure starts with a guess for the wave functions, and not for the KS potential, at variance with the vLB procedure. Also in the case of the CV method, we have checked that the starting guess is not influencing the result of the minimization. Indeed, starting either from constant wave functions or from harmonic oscillator orbitals (with the usual $\hbar \omega=41 \mathrm{~A}^{-1 / 3} \mathrm{MeV}$ ), we obtain satisfactory results.

The optimization of Eq. (15) is performed employing the IPOPT library [46,47]. Two main conditions determine the convergence of the algorithm:

(1) The relative tolerance on the violation of the constraints. This means that, at each step during the optimization, there is a test of the condition

$$
\max _{i}\left|\frac{g_{i}-c_{i}}{g_{i}}\right|<\epsilon,
$$

where the constraints are denoted by $c_{i}$, and the quantities subject to the constraints are denoted by $g_{i}$.

(2) The tolerance on the value of the objective function $f$. The algorithm stops if the change in value of the objective function between two successive iterations $k$ and $k-1$ is smaller than a given tolerance $\delta$, namely

$$
\left|f^{(k)}-f^{(k-1)}\right|<\delta
$$

Notice that while the $\mathrm{CV}$ method checks the convergence of the objective function $f$ [Eq. (19)], that is, of the rescaled radial wave functions $u_{i}(r)$, the convergence criterion of the vLB method [Eq. (11)] is based on the change of the KS potential $U[\rho]$.

The transformation in Eq. (17), that ensures numerical stability and accuracy in the solution of Eq. (15), has the effect that the Lagrange multipliers obtained with IPOPT have lost their direct and clear connection with $U(\vec{r})$ and $\varepsilon_{i j}$. Therefore, in a second step, we have written explicitly the differential equations that correspond to the variation of the cost functional. Since at this stage the auxiliary wave functions at the constrained minimum are known, the equations become a set of algebraic equations that is easily solved to obtain $U(\vec{r})$ and $\varepsilon_{i j}$ (cf. Ref. [19]).

\section{TEST OF THE NUMERICAL METHODS}

In this section, we test the two methods described in Sec. III by using target densities produced by Hartree-Fock (HF) calculations in the doubly magic nuclei ${ }^{40} \mathrm{Ca}$ and ${ }^{208} \mathrm{~Pb}$. The HF calculations have been carried out by using the Skyrme interaction SkX [48]. In the case of the Skyrme interactions the HF equations differ from the direct $\mathrm{KS}$ equations for the presence of the spin-orbit potential and of the effective mass $m^{*}(r)$. The nonlocalities introduced through effective masses are very mild in the case of the SkX interaction. For instance, the ratio $m^{*} / m$ in ${ }^{208} \mathrm{~Pb}$ lies between 0.92 and 1 and between 1 and 1.08, for protons and neutrons respectively. We then expect that the inverse KS algorithms lead to potentials which are similar to the SkX-HF potentials.

In Fig. 1, the neutron (left panels) and the proton (right panels) target densities from the HF calculations (black 

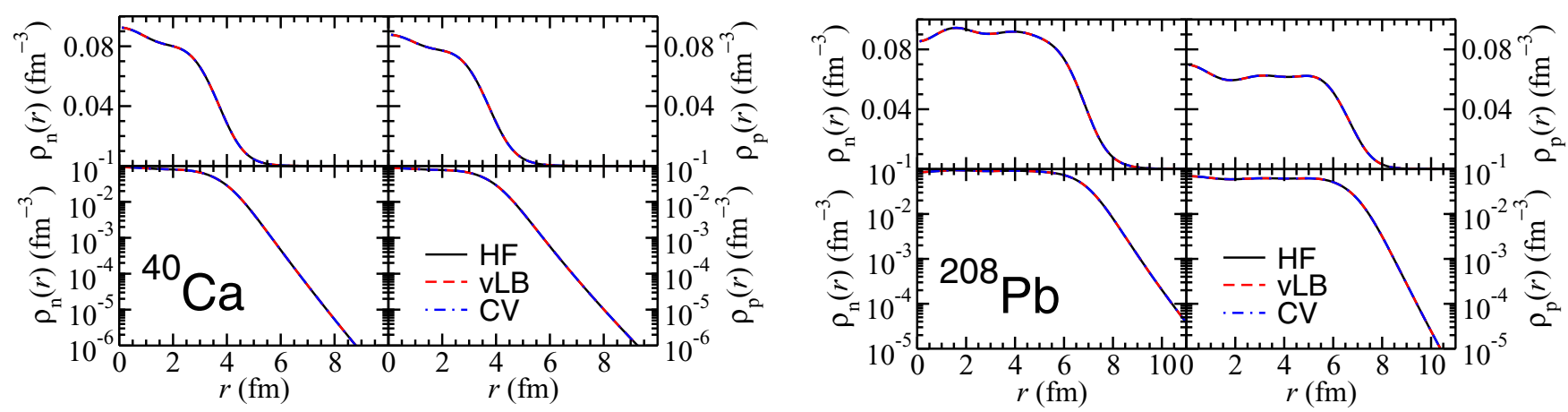

FIG. 1. Left figure: The neutron density, $\rho_{n}(r)$, and the proton density, $\rho_{p}(r)$, in ${ }^{40} \mathrm{Ca}$ are displayed as a function of the radial coordinate, in linear scale (top panels) and in logarithmic scale (bottom panels). The target densities obtained from HF calculations based on the SkX functional [48] (black solid line) are compared with the densities resulting from the two inversion methods discussed in the main text, namely vLB (red dashed line) and CV (blue dot-dashed line). Right figure: The same for the nucleus ${ }^{208} \mathrm{~Pb}$.

solid lines) in ${ }^{40} \mathrm{Ca}$ (left figure) and ${ }^{208} \mathrm{~Pb}$ (right figure) are shown. Upper panels show the different densities in a linear scale while the lower panels show the same quantities in a logarithmic scale. The logarithmic scale is important to check the asymptotics of the densities. The results of the inversion methods vLB (red dashed lines) and CV (blue dash-dotted lines) reproduce in very much detail the target HF densities. The maximum and average values (with respect to the radial coordinate) of the absolute differences are shown in Table I. In short, the reproduction of the target densities is fully satisfactory, for both neutrons and protons, with either method, in these two doubly magic nuclei. Nevertheless, the convergence criteria in the $\mathrm{CV}$ method are more restrictive in these calculations, as is evident from the same table.

The Kohn-Sham potentials obtained with the two inversion methods are shown in Fig. 2. As mentioned, the potentials are obtained up to an arbitrary constant. As explained in Sec. II, it is enough to shift the potential obtained through IKS so that the last occupied KS eigenvalue coincides with the last occupied HF eigenvalue to obtain such a constant.

Despite numerical errors, the results for the effective potentials appear to be rather satisfactory. The absolute value of the resulting difference $\Delta U$ with respect to the HF potentials is smaller than $2.5 \mathrm{MeV}$ both for protons and neutrons (cf. the upper panels in Fig. 2). This appears to be a quite reasonable agreement. We note that again the $\mathrm{CV}$ method seems to perform slightly better than the vLB method. The reason stems from the different convergence criteria. The

TABLE I. Maximum (Max.) and average (Aver.) differences between the target $\mathrm{HF}(\mathrm{SkX})$ and $\mathrm{KS}$ neutron $(n)$ and proton $(p)$ densities from the two inversion methods for the cases of ${ }^{40} \mathrm{Ca}$ and ${ }^{208} \mathrm{~Pb}$. Numerical values are all in $10^{-6} \mathrm{fm}^{-3}$.

\begin{tabular}{lccccc}
\hline \hline & \multicolumn{2}{c}{ vLB } & & \multicolumn{2}{c}{ CV } \\
\cline { 2 - 3 } Nucleus & Max. & Aver. & & Max. & Aver. \\
\hline${ }^{40} \mathrm{Ca}(p)$ & 8.0 & 1.3 & 1.0 & 0.2 \\
${ }^{40} \mathrm{Ca}(n)$ & 8.7 & 1.4 & & 1.0 & 0.2 \\
${ }^{208} \mathrm{~Pb}(p)$ & 2.6 & 0.3 & & 0.4 & 0.1 \\
${ }^{208} \mathrm{~Pb}(n)$ & 8.7 & 3.6 & 7.5 & 2.1 \\
\hline \hline
\end{tabular}

spin-orbit energy splittings, which exist in HF and are not taken care of in our procedure, have been checked to have no special influence (it is well known that spin-orbit shifts do not affect wave functions and densities, as a rule). We recall here that there is another source of difference between the HF eigenvalues and the KS eigenvalues. While the former contain some effects due to the effective mass $m^{*} / m \lesssim 1$, the latter assumes $m^{*} / m=1$. Thus, we note a small deviation of the KS potentials in their asymptotic behavior [21] for $r \rightarrow \infty$.

We now focus on the convergence of the procedures. The two algorithms behave in a quite different way. As explained in Sec. III A, the vLB method iterates the potential according to Eq. (10) and stops when the condition (11) is satisfied; in the present case, we set $\alpha=20 \mathrm{keV}$, and the iteration procedure is stopped when $\Delta U^{(k)} \leqslant 20 \mathrm{keV}$. In Fig. 3, we display the evolution of the quantity $\Delta U^{(k)}$ as a function of the number of iterations for the case of the neutrons in ${ }^{208} \mathrm{~Pb}$, on a linear scale (left panel) and on a logarithmic scale (right panel). For the sake of clarity, only a representative point every 150 iterations is shown. The values of $\Delta U^{(k)}$ obtained with the vLB method (shown by red diamonds) decrease rapidly from 1 to about $10^{-1}$ during the first 500 iterations; subsequently, the decrease continues but at a much slower pace. The results from the CV method (shown by blue dots) should be seen under a different light. The procedure does not use the quantity $\Delta U^{(k)}$ as a convergence criterion, but attempts to minimize the value of the kinetic energy taking into account the tolerance with which constraints should be fulfilled. Then, the values of $\Delta U^{(k)}$ corresponding to different iterations do not, and should not be expected to, decrease with a monotonic trend. They show instead an oscillatory behavior, which can be observed by looking at the right panel in logarithmic scale. With this caveat we nevertheless observe that the quantity $\Delta U^{(k)}$ shows an overall decreasing trend as the iteration process goes on, becoming small enough to conclude that the final result for the potential is indeed reliable.

\section{RESULTS FOR EXPERIMENTAL DENSITIES}

In this section, we extract KS potentials from the experimental densities. As case studies, we use the 

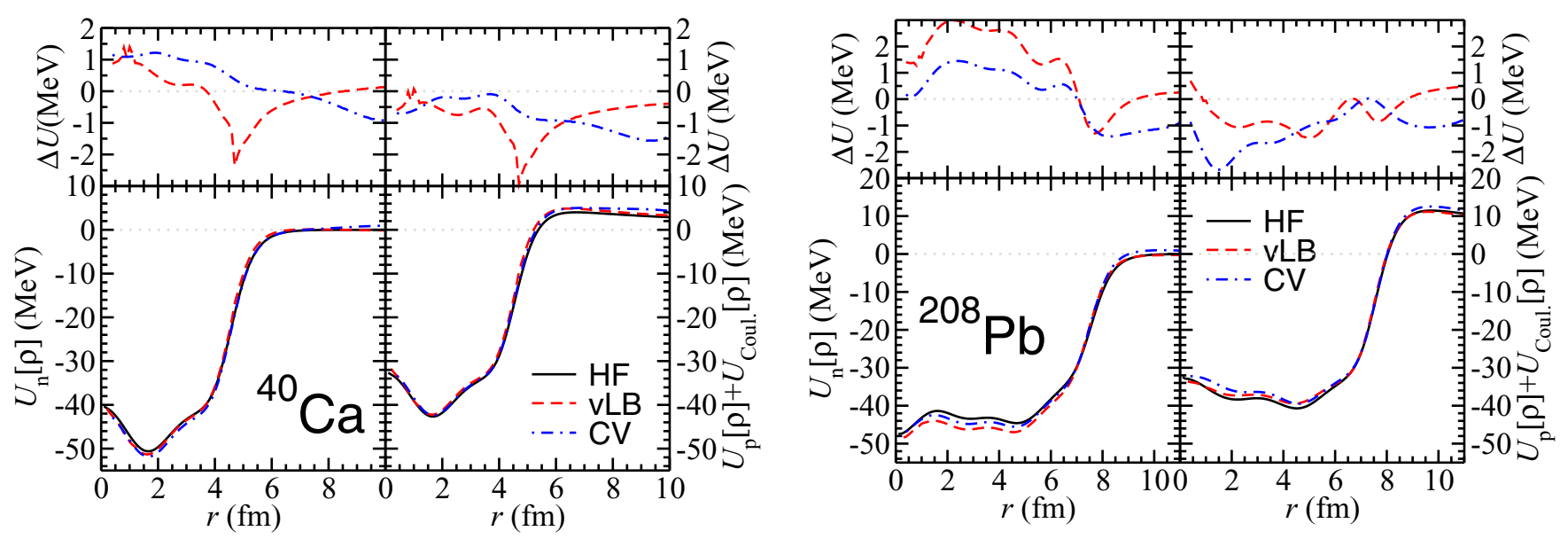

FIG. 2. Left figure: The Kohn-Sham potentials for neutrons and protons obtained with the vLB (red dashed lines) and CV (blue dot-dashed lines) inversion methods in ${ }^{40} \mathrm{Ca}$ are displayed in the bottom panels as a function of the radial coordinate. The benchmark HF calculations based on the SkX functional [48] are also shown (black solid lines). In the top panels, the differences $\Delta U \equiv U_{\text {method }}-U_{\mathrm{HF}}$ between the vLB and $\mathrm{CV}$ potential and the HF potential are shown. Right figure: The same, for the nucleus ${ }^{208} \mathrm{~Pb}$.

proton density of ${ }^{40} \mathrm{Ca}$ and the proton and neutron densities of ${ }^{208} \mathrm{~Pb}$. Specifically, the proton densities come from the electron scattering data of Ref. [49], while the neutron density of ${ }^{208} \mathrm{~Pb}$ has been extracted from proton scattering measurements in Ref. [50]. In both references, a parametrization of the electromagnetic charge and neutron densities based on a sum of Gaussian functions (SoG) can be found. This method was first introduced in Ref. [51] to extract nuclear charge densities from elastic electron scattering data without using model distributions but a basis of well behaved functions.
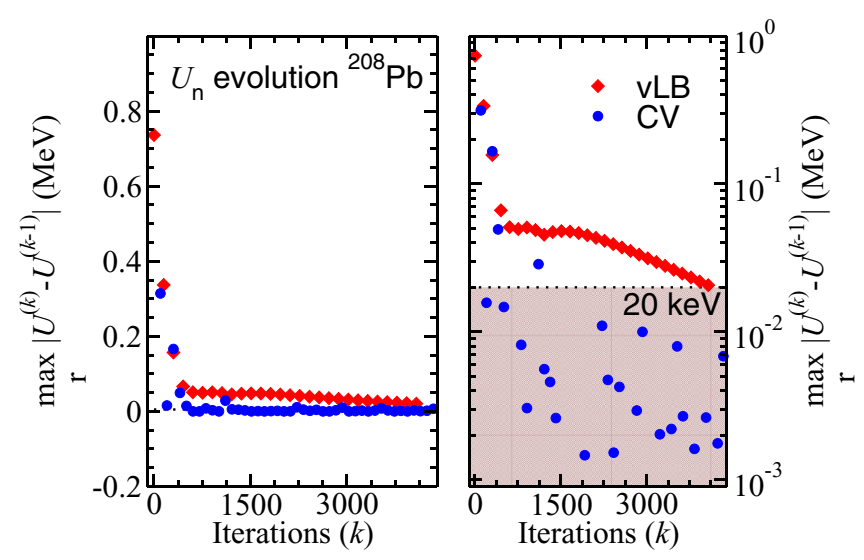

FIG. 3. Convergence test of the inversion methods, using as target density the results of a HF calculation with the SkX interaction. The absolute difference $\Delta U^{(k)}$ [cf. Eq. (11)] between the neutron Kohn-Sham potentials for ${ }^{208} \mathrm{~Pb}$ calculated at two successive iterations is shown at different iteration steps on a linear scale (left panel) and on a logarithmic scale (right panel). Results of the inversion method vLB and CV are shown by red diamonds and blue circles respectively. The value of $20 \mathrm{keV}$ associated with the convergence criterion of the vLB method, discussed in the main text, is highlighted.
The charge density distribution expressed as SoG can be written as

$$
\rho_{\text {charge }}(r)=\sum_{i} A_{i}^{\text {(charge })}\left(e^{-\left(\frac{r-R_{i}}{\gamma}\right)^{2}}+e^{-\left(\frac{r+R_{i}}{\gamma}\right)^{2}}\right) .
$$

The coefficients $A_{i}^{\text {(charge) }}$ are given by

$$
A_{i}^{\text {(charge) }}=\frac{Z e Q_{i}}{2 \pi^{\frac{3}{2}} \gamma^{3}\left(1+\frac{2 R_{i}^{2}}{\gamma^{2}}\right)},
$$

where $Q_{i}$ is the fraction of total charge that is associated with the integral of the $i$ th Gaussian. Accordingly, the normalization condition $\sum_{i} Q_{i}=1$ holds. The Gaussians are centered at different points $R_{i}$, whereas the widths are characterized by a common value $\gamma$. Sometimes, the value of $\gamma$ is taken to be close to the width of the narrowest peak that one finds when inspecting the square of typical Hartree-Fock or Woods-Saxon wave functions for the nucleus under study. The reason why the SoG parametrization (20) is chosen is that, if the sum contains enough terms, it corresponds to a model-independent representation of the actual data points. In principle, this would require a very large number of Gaussian terms if the experimental data could cover the full momentum transfer range, that is from 0 to infinity. In practice this is not the case and, thus, a manageable number of terms, of the order of $10-15$, has been proved to be stable against small changes. As can be easily understood, this representation may suffer from the fact that experimental data is scarce or does not cover a wide enough range of beam energies and scattering angles.

In order to extract the proton densities from the charge densities, we have neglected the small effects due to the electromagnetic spin-orbit and the neutron electromagnetic finite size (see for example Sec. II B of Ref. [52]). Hence, we have extracted the proton densities from the charge densities as

$$
\rho_{\text {charge }}(\vec{r})=\int d^{3} r^{\prime} f\left(\vec{r}^{\prime}\right) \rho_{p}\left(\vec{r}-\vec{r}^{\prime}\right)
$$




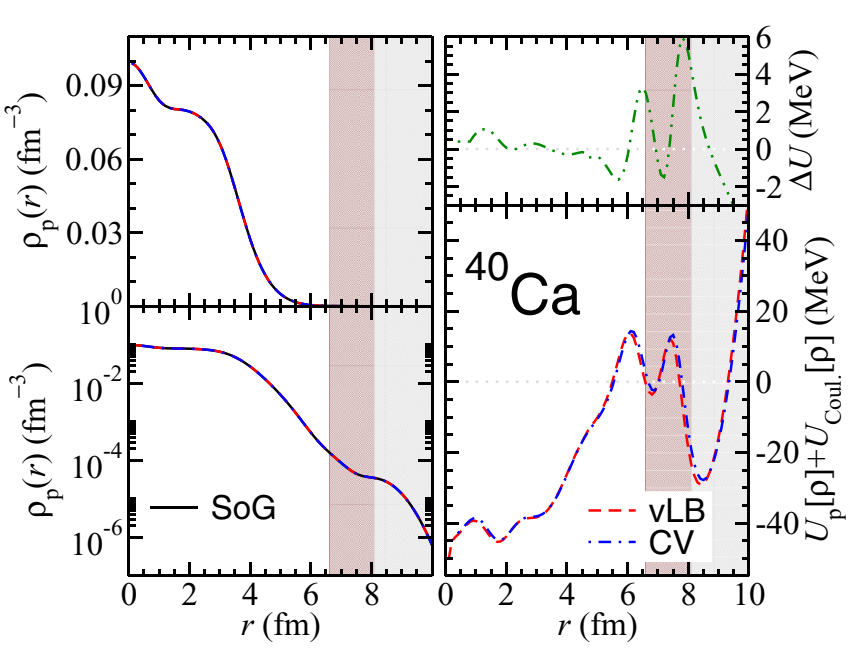

FIG. 4. The proton density for the case of ${ }^{40} \mathrm{Ca}$ is displayed as a function of the radial coordinate on a linear scale (top left panel) and on a logarithmic scale (bottom left panel). The target experimental density [49] labeled SoG_-sum of Gaussians_-(black solid lines) is compared with those obtained with the inversion methods vLB (red dashed lines) and CV (blue dot-dashed lines). In the bottom right panel, the Kohn-Sham potentials obtained within the two inversion methods are compared, and in the top right panel their difference $\Delta U=U_{\mathrm{CV}}-U_{\mathrm{vLB}}$ is shown.

using the approximate electric proton form factor

$$
f(\vec{r})=\frac{q_{e}}{\pi^{3 / 2} \alpha^{3}} e^{-\left(\frac{r}{\alpha}\right)^{2}},
$$

where $q_{e}$ is the proton charge, $\alpha$ has been taken as $\sqrt{2 / 3} R_{p}$ and the value $R_{p}=0.87 \mathrm{fm}$ has been assumed for the r.m.s. proton radius. Small changes to the chosen value for $R_{p}$ will not appreciably change our results. The deconvolution that leads to the proton charge density is performed using the regular product in Fourier space. Due to the properties of the Gaussian functions, the result in coordinate space can be analytically written assuming spherical symmetry as

$$
\begin{aligned}
\rho_{p}(r)= & \sum_{i} \frac{\gamma^{3} A_{i}}{e \beta r}\left[\left(\frac{r-R_{i}}{\beta^{2}}+\frac{R_{i}}{\gamma^{2}}\right) e^{-\left(\frac{r-R_{i}}{\beta}\right)^{2}}\right. \\
& \left.+\left(\frac{r+R_{i}}{\beta^{2}}-\frac{R_{i}}{\gamma^{2}}\right) e^{-\left(\frac{r+R_{i}}{\beta}\right)^{2}}\right],
\end{aligned}
$$

where $\beta=\sqrt{\gamma^{2}-\alpha^{2}}$.

In the case of neutrons, such a procedure is not needed, as Ref. [50] provides the neutron density in the form of Eq. (20) directly. These data have been obtained via proton elastic scattering. Protons interact via the strong interaction with both neutrons and protons. So if the proton density is known, one can derive the neutron density compatible with the experimental cross section. This procedure is not model independent, at variance with the case of electron elastic scattering used to determine the electromagnetic charge density. In fact, the proton-nucleus interaction at intermediate incident energy is relatively well known but has some uncertainty.

We have implemented the same procedure described in Sec. III in order to solve the IKS problem with the input of the experimental densities. We have converged to KS densities that display a good agreement with the experimental densities. The agreement can be seen in the left panels of Fig. 4 for

${ }^{40} \mathrm{Ca}$ and Fig. 5 for ${ }^{208} \mathrm{~Pb}$. The relative differences found for the densities are of the same order of those found in the previous section. Since the differences between the vLB and $\mathrm{CV}$ densities and the target densities are not visible in detail from the figures, the maximum and the average of the absolute value of these differences are reported in Table II.
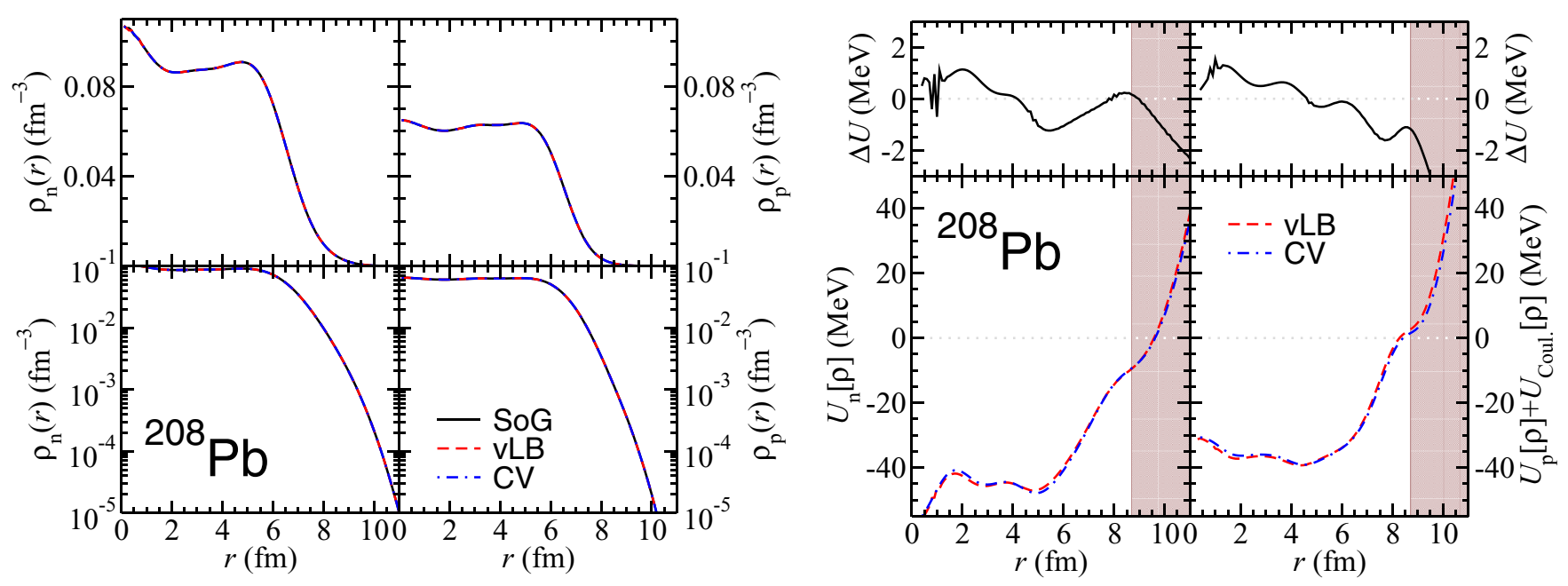

FIG. 5. Left figure: The neutron and proton densities (left panels) for the case of ${ }^{208} \mathrm{~Pb}$ are displayed as a function of the radial coordinate, on a linear scale (top panels) and on a logarithmic scale (bottom panels). The target experimental densities [49] labeled as SoG-sum of Gaussians-(black solid lines) are compared with those obtained with the inversion methods vLB (red dashed lines) and CV (blue dot-dashed lines). Right figure: The Kohn-Sham potentials calculated for neutrons and protons with the inversion methods vLB (red dashed lines) and CV (blue dot-dashed lines) are shown. In the top panels, the corresponding differences between the Kohn-Sham potentials $\Delta U=U_{\mathrm{CV}}-U_{\mathrm{vLB}}$ are displayed. 
TABLE II. Maximum (Max.) and average (Aver.) differences between the target experimental (SoG) and KS neutron $(n)$ and proton $(p)$ densities from the two inversion methods for the cases of ${ }^{40} \mathrm{Ca}$ and ${ }^{208} \mathrm{~Pb}$. Numerical values are in $10^{-6} \mathrm{fm}^{-3}$.

\begin{tabular}{lrrrrr}
\hline \hline & \multicolumn{2}{c}{ vLB } & & \multicolumn{2}{c}{$\mathrm{CV}$} \\
\cline { 2 - 3 } \cline { 6 - 6 } Nucleus & Max. & Aver. & & Max. & Aver. \\
\hline${ }^{40} \mathrm{Ca}(p)$ & 14.5 & 1.6 & & 0.9 & 0.2 \\
${ }^{208} \mathrm{~Pb}(p)$ & 5.0 & 1.1 & & 0.2 & 0.04 \\
${ }^{208} \mathrm{~Pb}(n)$ & 17.1 & 1.3 & & 0.4 & 0.1 \\
\hline \hline
\end{tabular}

The Kohn-Sham potentials, shifted by using the experimental neutron and proton separation energies, obtained with the vLB and CV methods, are also shown in Fig. 4 for ${ }^{40} \mathrm{Ca}$ and Fig. 5 for ${ }^{208} \mathrm{~Pb}$. The agreement between the two inversion methods is remarkable and of the same quality as that found in Sec. IV for the HF test cases (see the right panels in Figs. 4 and 5). However, while the potentials in the inner part of the nuclei look very reasonable, they oscillate and then tend to increase without limit in the asymptotic region. That is, the asymptotic behavior of the KS potentials at large distances is not the expected one. This has to be attributed to the Gaussian tail of the SoG density that both algorithms translate into a quadratic (i.e., harmonic-oscillator-like) potential. To substantiate this interpretation, the regions corresponding to $r$ larger than the radius of the outermost (second outermost) Gaussian in the case of ${ }^{208} \mathrm{~Pb}\left({ }^{40} \mathrm{Ca}\right)$ are shown as shadowed areas in Figs. 4 and 5, respectively. The borders of these regions are clearly correlated with the change in slope of the potentials. As a consequence, our results for the experimentally derived KS potentials cannot be regarded as reliable in the tail of the potential.

We can conclude that the employed inversion procedure remains robust when experimental SoG densities are input and provides us with reliable information about the potential, except for its tail.

\section{CONCLUSIONS AND PERSPECTIVES}

We have addressed the inverse Kohn-Sham problem in the case of the atomic nucleus for the first time, employing two well-known inversion methods that have been used in other fields in physics [19]. The first method is based on an iterative procedure [23] (vLB) and the second consists of a constrained minimization of the kinetic energy [19] (CV). We have applied the inversion methods on the two closed shell, spherical nuclei ${ }^{40} \mathrm{Ca}$ and ${ }^{208} \mathrm{~Pb}$. We first tested the numerical algorithms, deriving the KS potential from a density obtained by a HF calculation with a Skyrme interaction characterized by an effective mass close to the bare mass. We verified that the resulting KS potential is in good agreement with the HF potential. We then applied the inversion methods to the experimentally derived densities of protons in ${ }^{40} \mathrm{Ca}$ and ${ }^{208} \mathrm{~Pb}$ and neutrons in ${ }^{208} \mathrm{~Pb}$. The consistency between vLB and CV remains remarkable, and the potentials obtained in the interior and at the surface of the nucleus appear to be reliable. On the other hand, the parametrization of the experimental density as a sum of Gaussians ( $\mathrm{SoG}$ ) used in this work leads to difficulties in the tails of the potentials. The nonphysical Gaussian tails probed by the algorithm at large distances translate into harmonic-oscillator-like potentials that diverge.

(i) Although attempting to use the outlined procedure for a larger set of nuclei, including deformed ones, might be of interest, it is quite clear from the start that the mere experimental information about neutron and proton densities is insufficient to deduce an effective KS potential. In fact, as we mentioned in the text above, we know that a realistic nuclear EDF depends also on gradients of the densities, spin densities and their combinations. These cannot be experimentally determined, and we have to rely on ab initio calculations. As $a b$ initio nuclear structure is progressing, the first step to be undertaken should be to test the IKS when densities from $a b$ initio calculations are input. This will allow one to formulate the IKS scheme in a somehow more general manner. As we mentioned in the text above, we envisage proceeding along at least two directions. We will try to fix the equation of state (EoS) of uniform matter directly from $a b$ initio calculations, and formulate the IKS for finite nuclei in such a way that only the gradient terms need to be extracted. At the same time, we will try to use the spin densities from $a b$ initio calculations to extract the spin part of the effective potential. We are fully aware that in principle other kinds of densities should enter the analysis, and further progress will eventually be needed; however, at this first stage, we will stick to gradual steps and take, for instance, the spin-orbit and Coulomb parts of the EDF as uncorrelated [13].

(ii) A specific aspect, yet related to the previous point, is the issue of locality. There is no guarantee that a purely local effective potential is the correct choice. In nuclear physics, local EDFs produce local potentials (as in the case of Skyrme EDFs) but nonlocal EDFs and nonlocal potentials also exist (as in the case of Gogny); however, there is a way to reparametrize nonlocality in terms of a power expansion in gradients, as shown in Ref. [53]. Nonlocal densities from ab initio calculations can be inspected to understand the degree of nonlocality that is needed.

(iii) Last but most importantly, extracting an effective potential from IKS is not enough to determine the quantity of real interest, that is the EDF itself. As shown by van Leeuwen and Baerends in Ref. [36], knowing the effective potential along a path of densities can give access to the EDF. In a few cases, we can expect that $a b$ initio calculations can explore systems that are very close to each other in terms of density distributions. One example is neutron drops (see [54,55] and references therein): systems of neutrons confined by a harmonic oscillator potential. We shall explore the possibility of other cases in which $a b$ initio calculations can be performed for systems whose densities define a continuous path. Last but not least, checking whether this idea is related to the one 
introduced in Ref. [56] is also to be considered a task to deal with.

Most likely, the most promising path to follow is probably the one in which the IKS is used in parallel with other techniques to derive an EDF ab initio, as a way to fine tune specific terms and not as a unique strategy. We envisage starting soon to apply the IKS method to densities from $a b$ initio approaches and to understand how the current scheme can be generalized.

\section{ACKNOWLEDGMENT}

Funding from the European Union's Horizon 2020 research and innovation programme under Grant Agreement No. 654002 is acknowledged.
[1] M. Bender, P.-H. Heenen, and P.-G. Reinhard, Rev. Mod. Phys. 75, 121 (2003).

[2] Energy Density Functional Methods for Atomic Nuclei, edited by N. Schunck (IOP, Bristol, 2019).

[3] K. Burke, J. Chem. Phys. 136, 150901 (2012).

[4] A. D. Becke, J. Chem. Phys. 140, 18A301 (2014).

[5] P. Hohenberg and W. Kohn, Phys. Rev. 136, B864 (1964).

[6] F. Raimondi, B. G. Carlsson, and J. Dobaczewski, Phys. Rev. C 83, 054311 (2011).

[7] P. Becker, D. Davesne, J. Meyer, J. Navarro, and A. Pastore, Phys. Rev. C 96, 044330 (2017).

[8] Y. Engel, D. Brink, K. Goeke, S. Krieger, and D. Vautherin, Nucl. Phys. A 249, 215 (1975).

[9] J. Dobaczewski and J. Dudek, Acta Phys. Pol. B 27, 45 (1996).

[10] E. Perlińska, S. G. Rohoziński, J. Dobaczewski, and W. Nazarewicz, Phys. Rev. C 69, 014316 (2004).

[11] J. Drut, R. Furnstahl, and L. Platter, Prog. Part. Nucl. Phys. 64, 120 (2010).

[12] L. G. Cao, U. Lombardo, C. W. Shen, and N. V. Giai, Phys. Rev. C 73, 014313 (2006).

[13] M. Baldo, P. Schuck, and X. Viñas, Phys. Lett. B 663, 390 (2008).

[14] D. Gambacurta, L. Li, G. Colò, U. Lombardo, N. Van Giai, and W. Zuo, Phys. Rev. C 84, 024301 (2011).

[15] M. Stoitsov, M. Kortelainen, S. K. Bogner, T. Duguet, R. J. Furnstahl, B. Gebremariam, and N. Schunck, Phys. Rev. C 82, 054307 (2010).

[16] S. K. Bogner, R. J. Furnstahl, H. Hergert, M. Kortelainen, P. Maris, M. Stoitsov, and J. P. Vary, Phys. Rev. C 84, 044306 (2011).

[17] R. Navarro Pérez, N. Schunck, A. Dyhdalo, R. J. Furnstahl, and S. K. Bogner, Phys. Rev. C 97, 054304 (2018).

[18] W. Kohn and L. J. Sham, Phys. Rev. 140, A1133 (1965).

[19] D. S. Jensen and A. Wasserman, Int. J. Quantum Chem. 118, e25425 (2018).

[20] J. P. Perdew, R. G. Parr, M. Levy, and J. L. Balduz, Phys. Rev. Lett. 49, 1691 (1982).

[21] J. P. Perdew and M. Levy, Phys. Rev. B 56, 16021 (1997).

[22] Y. Wang and R. G. Parr, Phys. Rev. A 47, R1591 (1993).

[23] R. van Leeuwen and E. J. Baerends, Phys. Rev. A 49, 2421 (1994).

[24] E. A. Hylleraas, Z. Phys. 54, 347 (1929).

[25] J. Li, N. D. Drummond, P. Schuck, and V. Olevano, SciPost Phys. 6, 40 (2019).

[26] S. E. B. Nielsen, M. Ruggenthaler, and R. van Leeuwen, Europhys. Lett. 101, 33001 (2013).

[27] S. E. B. Nielsen, M. Ruggenthaler, and R. van Leeuwen, Eur. Phys. J. B 91, 235 (2018).

[28] A. Kumar, R. Singh, and M. K. Harbola, J. Phys. B: At. Mol. Opt. Phys. 52, 075007 (2019).

[29] B. Kanungo, P. M. Zimmerman, and V. Gavini, Nat. Commun. 10, 4497 (2019).
[30] T. Naito, D. Ohashi, and H. Liang, J. Phys. B: At. Mol. Opt. Phys. 52, 245003 (2019).

[31] A. S. Umar and V. E. Oberacker, Phys. Rev. C 74, 021601(R) (2006).

[32] J. Hadamard, Princeton Univ. Bull. 13, 49 (1902).

[33] J. T. Chayes, L. Chayes, and M. B. Ruskai, J. Stat. Phys. 38, 497 (1985).

[34] R. M. Dreizler and E. K. U. Gross, Density Functional Theory: An Approach to the Quantum Many-Body Problem (Springer, Berlin, 1990).

[35] A. P. Gaiduk, S. K. Chulkov, and V. N. Staroverov, J. Chem. Theory Comput. 5, 699 (2009).

[36] R. van Leeuwen and E. J. Baerends, Phys. Rev. A 51, 170 (1995).

[37] J. Engel, Phys. Rev. C 75, 014306 (2007).

[38] M. Valiev and G. W. Fernando, arXiv:cond-mat/9702247.

[39] G. W. Fernando, in Metallic Multilayers and Their Applications, edited by G. W. Fernando, Handbook of Metal Physics Vol. 4 (Elsevier, Amsterdam, 2008), pp. 131-156.

[40] N. Barnea, Phys. Rev. C 76, 067302 (2007).

[41] J. Messud, M. Bender, and E. Suraud, Phys. Rev. C 80, 054314 (2009).

[42] C. Titin-Schnaider and P. Quentin, Phys. Lett. B 49, 397 (1974).

[43] X. Roca-Maza, L.-G. Cao, G. Colò, and H. Sagawa, Phys. Rev. C 94, 044313 (2016).

[44] Q. Wu and W. Yang, J. Chem. Phys. 118, 2498 (2003).

[45] R. Parr and W. Yang, Density-Functional Theory of Atoms and Molecules, International Series of Monographs on Chemistry (Oxford University, New York, 1994).

[46] A. Wächter and L. T. Biegler, Math. Program. 106, 25 (2006).

[47] A. Wächter, Short tutorial: getting started with ipopt in 90 minutes, in Combinatorial Scientific Computing 2009, edited by U. Naumann, O. Schenk, H. D. Simon, and S. Toledo, Dagstuhl Seminar Proceedings (Schloss Dagstuhl - Leibniz-Zentrum fuer Informatik, Germany), https://drops.dagstuhl.de/opus/volltexte/ 2009/2089/.

[48] B. A. Brown, Phys. Rev. C 58, 220 (1998).

[49] H. D. Vries, C. D. Jager, and C. D. Vries, At. Data Nucl. Data Tables 36, 495 (1987).

[50] J. Zenihiro, H. Sakaguchi, T. Murakami, M. Yosoi, Y. Yasuda, S. Terashima, Y. Iwao, H. Takeda, M. Itoh, H. P. Yoshida, and M. Uchida, Phys. Rev. C 82, 044611 (2010).

[51] I. Sick, Nucl. Phys. A 218, 509 (1974).

[52] L. Ray, Phys. Rev. C 19, 1855 (1979).

[53] B. G. Carlsson and J. Dobaczewski, Phys. Rev. Lett. 105, 122501 (2010).

[54] P. W. Zhao and S. Gandolfi, Phys. Rev. C 94, 041302(R) (2016).

[55] S. Shen, H. Liang, J. Meng, P. Ring, and S. Zhang, Phys. Rev. C 97, 054312 (2018).

[56] J. Dobaczewski, J. Phys. G: Nucl. Part. Phys. 43, 04LT01 (2016). 